\title{
Biotelemetry recording of the electrical activity of the hippocampus and amygdala during sexual behavior in the cat
}

\author{
THOMAS L. BENNETT, PAULA L. HILL, and JONATHAN FRENCH \\ Colorado State University, Fort Collins, Colorado 80523
}

\begin{abstract}
Five male and five female cats were anesthetized, and electrodes were implanted in the dorsal hippocampus and the basolateral amygdala. After recovery, a paired female and male were placed in a large playroom in which recordings were taken via biotelemetry during sexual behavior. Each session lasted $4 \mathrm{~h}$ or four successful matings, whichever came first. Both amygdala $40-\mathrm{Hz}$ activity and hippocampal rhythmic slow-wave activity (RSA) of 4-5 $\mathrm{Hz}$ were very prominent during sexual behavior. Hippocampal RSA was the dominant activity in both male and female cats during all components of sexual behavior except pelvic thrusts just prior to intromission, when hippocampal desynchronization dominated the records. Amygdala $40-\mathrm{Hz}$ activity was the dominant activity in all female and three male cats during all aspects of sexual behavior. Two male cats showed no $40-\mathrm{Hz}$ activity from the point of neck grip until release after intromission, presumably due to the interruption of airflow through nasal passages. The results are discussed in relation to contemporary theories regarding the behavioral correlates of hippocampal RSA and amygdala 40-Hz activity.
\end{abstract}

During the past 25 years, a number of researchers have attempted to correlate different electroencephalographic (EEG) patterns recorded from the hippocampus and the amygdala to specific behavioral processes in the hope that such endeavors would clarify the functions of these structures. Analysis of the bioelectric rhythms of the hippocampus has focused on the possible significance of its high-amplitude rhythmic slow-wave activity (RSA) $(4-12 \mathrm{~Hz})$, which has been related by different theorists to functions performed by this structure in arousal (Green \& Arduini, 1954), attention and information processing (Bennett, 1975), learning and memory (Adey, 1966), and voluntary movement (Vanderwolf, Kramis, Gillespie, \& Bland, 1975). The second major pattern of the hippocampus, a low-amplitude, irregular, faster frequency response, is called hippocampal desynchronization. Although desynchronization has not received as much attention in the hippocampal literature, its significance has been addressed by some researchers. Vanderwolf and his colleagues suggest that it may reflect hippocampal regulation of automatic or reflexive behaviors. Bennett (1979) has recently proposed that desynchronization reflects an active function of the hippocampus in filtering or gating out irrelevant or redundant environmental information.

Interest in the electrical activity of the amygdala has been directed toward understanding the significance of

Requests for reprints should be sent to Thomas L. Bennett, Department of Psychology, Colorado State University, Fort Collins, Colorado 80523. The current address of Jonathan French is Department of Neurology, Cornell Medical Center, 525 East 68th Street, New York, New York 10021. amygdala fast activity. Amygdala fast activity, which is most easily observed using electrodes placed in the basolateral complex of this structure, refers to bursts of fast, rhythmic activity that appear against a background of desynchronized activity. The dominant frequency of these bursts is $40 \mathrm{~Hz}$. Amygdala fast activity is a correlate of olfactory orienting responses (Bennett \& French, 1977; Delgado, Johnston, Wallace, \& Bradley, 1970). This EEG pattern is highly correlated with sniffing, and it is dependent on the movement of air through the nasal passages (Gault \& Leaton, 1963; Sheer, 1970).

The electrical activity of the hippocampus and/or the amygdala has been recorded from chronically implanted animals in a variety of behavioral situations. Several inquiries, including the present one, have used copulatory behavior as the con text in which to investigate these bioelectric responses. The advantage of using sexual behavior is that in the rat and cat (the species used in these experiments), it is a stable, naturally occurring behavior pattern, and it can be subdivided into voluntary goal-directed behaviors, au tomatic stereotyped responses, and consummatory acts (Kurtz \& Adler, 1973). Kurtz (1975; Kurtz \& Adler, 1973) has published extensive observations of the hippocampal EEG correlates of components of sexual behavior in the male and female rat. She suggested that high-frequency RSA $(8-9 \mathrm{~Hz})$ is associated with sexual appetitive behaviors and slower frequency RSA $(7-8 \mathrm{~Hz})$ accompanies sexual consummatory responses.

The electrical activity of the amygdala during copulation in cats has received limited study. Schwartz and Whalen (1965) reported that $40-\mathrm{Hz}$ activity became more prominent and increased in amplitude during intro- 
mission, as compared with preintromission, in the two male cats they observed. Holmes and Egan (1973) reported that the female cat's amygdala fast activity showed a dramatic increase in the amplitude of this highfrequency rhythm immediately upon intromission; in most instances, the amount of $40-\mathrm{Hz}$ activity per unit of time increased as well. This bioelectric response persisted throughout the female's afterreaction (a series of violent rolls after copulation). Holmes and Egan observed bursts of $40-\mathrm{Hz}$ activity in their male cats throughout the mating performance. However, in contrast to their female cats and to the male data reported by Schwartz and Whalen, there was no increase in the amplitudes of the $40-\mathrm{Hz}$ rhythm during intromission.

The present study had a threefold purpose. The first was to replicate and extend the work of Holmes and Egan (1973) and Schwartz and Whalen (1965), that is, to further study the activity of the amygdala during sexual behavior. The second was to examine in detail the different components of the cat's sexual behavior and to correlate the behavior with hippocampal electrical activity, as was done by Kurtz (1975) in rats. The third purpose was to investigate the relationships between the amygdala and hippocampal rhythms during each component of sexual behavior.

\section{METHOD}

\section{Subjects}

The subjects were 10 adult cats, 5 sexually intact males and 5 ovariectomized females, with bipolar electrodes bilaterally implanted in the dorsal hippocampus and basolateral amygdala. The stereotaxic coordinates were the same as those used by Bennett and French (1977). The accuracy of the placements was verified histologically.

\section{Procedure}

During observations of sexual behavior, the EEG data from the hippocampus and the amygdala were transmitted from the animal via two telemetry transmitters (Narco FM 1100-02). A leather harness held the transmitters securely on the cat's back. The signal was received by a Narco biotelemetry receiver (FM 1100-07) that interfaced with the preamplifier of a Grass Model 7B polygraph, and a paper record of the signals was obtained for visual analysis. The EEG data from one hippocampal and one amygdala electrode were recorded simultaneously with verbal description of the subject's activities on a Data Inc. Model 1400 FM tape recorder.

The subjects were observed in a room that was $1.88 \times 2.67$ $\mathrm{x} 2.72 \mathrm{~m}$ and contained carpeted ramps, shelves, and a pan of kitty litter. Observation of the entire testing room from an adjacent darkened room was made through a Plexiglas observation window, measuring $77.57 \times 77.57 \mathrm{~cm}$. The biotelemetry receiver and polygraph were also located in the darkened room.

Each session involved a paired female and male and lasted $4 \mathrm{~h}$ or until four or more successful matings, whichever came first. A single stimulus male was used to mate with all of the females and a single stimulus female was used to mate with all of the males. The male was introduced into the testing room approximately $15 \mathrm{~min}$ before the female. Acceptability of the male by the female was the criterion used to determine if the female was in true estrus.

Hormone injection. The females were brought into estrus by a subcutaneous injection of either estradiol in aqueous solution $(1 \mathrm{mg} / \mathrm{cc})$ or estradiol cyprenate $(2 \mathrm{mg} / \mathrm{ml})$. The estradiol injection regimen involved daily .1-mg injections until the onset of estrus; subsequently, a maintenance dose of the same amount was administered every other day until the experiment ended. The estradiol cyprenate regimen involved an injection of $.3 \mathrm{mg}$ on the 1 st day and a subsequent dose $48 \mathrm{~h}$ later that brought the female into estrus. If the experiment ran an unusually long period of time (more than 10 days), an additional dose of estradiol cyprenate was required to maintain estrus. The subjects were neither food nor water deprived during the experiment.

Assessment of behavior. In the present study, the following sequence of events, as previously documented by Michael (1961), was observed and recorded when the female was sexually receptive: The female bent down with her chest and abdomen extremely close to the floor, and her head was held down between her extended front paws; her back legs were partially extended, elevating her pelvic area (lordosis). The tail moved to the side to expose her perineum. The back legs moved in an alternate treading pattern. During lordosis, the male mounted her and obtained a neck grip with his jaws. The female's treading then became more rapid, and the pair began to move backward. The backward movement and treading coincided with the male's copulatory thrusts. These thrusts produced a greater degree of pelvic elevation in the female, so that the perineum was turned backward and upward. Intromission could occur during one of these movements. When intromission occurred, the female emitted a loud piercing cry, and within a matter of seconds, she jumped away from the male. She then licked herself all over and began a series of violent rolls (the afterreaction). The male remained a few feet away and watched the female during the afterreaction. The afterreaction gradually disappeared, and in about 10-20 $\mathrm{min}$, the female was again receptive.

Analysis of EEG data. The data were visually analyzed, and the analysis correlated the discussion of behavior with the EEG tracings that were obtained on magnetic tape as a permanent record. The analysis determined the dominant EEG pattern shown in the hippocampus and the amygdala during each specific segment of sexual behavior. The patterns were scored as hippocampal RSA ( a train of RSA), hippocampal desynchrony, amygdala fast activity $(40 \mathrm{~Hz})$, or amygdala desynchrony. The frequency of an EEG pattern was determined visually by counting the peaks in a $1 \mathrm{sec}$ time period. There were seven sexual behaviors observed in the females and five in the males. The behaviors observed in the female were reaction to neck grip by the male, reaction to mount by the male, lordosis, treading of the back legs, reaction to the male's pelvic thrusts, intromission, and afterreaction. The behaviors observed in the male were neck grip, mount, pelvic thrust, intromission, and observation of the female's afterreaction.

\section{RESULTS}

During this 6-month study, usable EEG data were collected from 51 successful matings, 27 from the males and 24 from the females. A mating was considered successful when the female emitted a loud cry during intromission and proceeded to show an afterreaction. At least four different matings were recorded for each animal. During the matings, all of the female and male sexual behaviors described previously were observed. During each session, each phase of the mating process for both male and female was examined and correlated with the EEG recordings of the hippocampus and amygdala.

\section{Hippocampal Activity}

In the hippocampus, RSA in the range of $4-5 \mathrm{~Hz}$ was the dominant bioelectric rhythm during all but one of 
the sexual behaviors in the male. As the female entered the room, RSA became very prominent in all the males. This pattern of electrical activity continued during the male's preliminary exploration of the female. At the point of the neck grip and during the mount, 4 to $5-\mathrm{Hz}$ RSA continued to be the dominant activity. As pelvic thrusting began, however, desynchronized activity became more prominent in the hippocampus, and just before intromission, hippocampal desynchronization became dominant. During intromission, 4 to $5-\mathrm{Hz}$ RSA reappeared, and this rhythm continued to dominate the electrical activity of the hippocampus as the male observed the female during the afterreaction. It was during this phase of sexual behavior that RSA was most prominent. In all males, RSA occurred whether the subject was walking around and watching the female or sitting and staring at her.

In the female, 4- to $5-\mathrm{Hz}$ RSA was prominent as she entered the room and became acquainted with the male through mutual exploration. Subsequently, the male approached the female, secured a neck grip, and mounted her. During this interval, RSA of $4-5 \mathrm{~Hz}$ was dominant. Throughout neck grip and mount, the female was in lordosis and treading her back legs. Even though RSA was present throughout the period prior to intromission, RSA did not appear to be as prominent as it was in the male during the same interval. Just before intromission, during vigorous pelvic thrusting by the male, the occurrence of RSA decreased and desynchronized activity became dominant. At the point of intromission, which is accompanied by a loud cry, RSA again became the dominant activity in four of the five females. RSA appeared to continue at a high amplitude thoughout the afterreaction, but movement artifact often obscured the EEG signals. As the afterreaction subsided and the rest period began, the amplitude returned to preintromission levels.

\section{Amygdala Fast Activity}

Bursts of amygdala $40-\mathrm{Hz}$ activity were observed in both the females and males at the onset of each session and during preliminary exploration. Three of the males also showed $40-\mathrm{Hz}$ activity during neck grip, mount, and pelvic thrust, but it disappeared during intromission. However, two males did not follow this pattern. As soon as these males secured a neck grip, $40 \cdot \mathrm{Hz}$ activity disappeared and did not reappear until the neck grip was released after intromission. During the observation of the female's afterreaction, the incidence of $40-\mathrm{Hz}$ activity returned to levels seen before neck grip in both groups.

The females showed $40-\mathrm{Hz}$ activity during the male's neck grip and mount and also during lordosis and treading. During pelvic thrusting by the male, however, desynchronized activity in the amygdala became dominant, especially just prior to intromission. At the time of intromission, the $40-\mathrm{Hz}$ activity returned and the amplitude increased to a level above preintromission level and remained there throughout the afterreaction. As the rest period began, the amplitude returned to preintromission level.

\section{DISCUSSION}

\section{Hippocampal Activity}

In the male, 4 to $5-\mathrm{Hz}$ hippocampal RSA was the dominant brain-wave pattern during approach, neck grip, mount, intromission, and observation of the female's afterreaction. 'During the period of pelvic thrusting, just prior to intromission, RSA was replaced by desynchronized activity as the dominant EEG pattern. In the female, hippocampal RSA activity was dominant during lordosis, treading, and intromission. As in the male, during pelvic thrusting and just prior to intromission, the incidence of RSA decreased and desynchronization became dominant. It was also apparent that RSA was less prominent in the female than in the male during the mating sequence. Movement artifact obscured the record during the afterreaction in the female, but RSA appeared to be the dominant bioelectric activity.

These findings are similar to Kurtz's (1975; Kurtz \& Adler, 1973) observations made in the rat, in that hippocampal RSA was the dominant pattern of electrical activity during male and female sexual behavior. However, aside from this general finding, there were several specific differences in our data. First, in the cat, we observed that hippocampal desynchronization was the dominant activity during pelvic thrusts; Kurtz observed that RSA was the dominant activity in rat hippocampal recordings during this same period. Immediately after intromission, male rat RSA was replaced by desynchronized activity. In the present study, the male cat hippocampal records changed from being dominated by desynchronized activity to RSA, an opposite outcome. Finally, we did not observe the changes in the frequency of the RSA response during appetitive vs. consummatory behavior that Kurtz reported. Instead, RSA of $4-5 \mathrm{~Hz}$ was the dominant pattern whenever RSA occurred. In fact, we have rarely observed RSA of greater than $6 \mathrm{~Hz}$ in the hippocampal records of cats (never over $7 \mathrm{~Hz}$ ).

The distinction between appetitive and consummatory components of sexual behavior that was reflected by different frequencies of RSA in Kurtz's (1975) observations with rats was not supported by the present findings. Similarly, since all aspects of sexual behavior, with the exception of pelvic thrusting, were accompanied by RSA in the cat, our findings do not support the view that voluntary vs. automatic behavior responses are accompanied by RSA vs. hippocampal desynchronization, respectively, as has been suggested by Vanderwolf et al. (1975). The most parsimonious interpretation of our data is that RSA was a correlate of the cats' attending to environmental cues (namely, each other) that would help them complete successfully the chain of events comprising copulation.

\section{Amygdala Fast Activity}

Amygdala $40-\mathrm{Hz}$ activity was observed in both male and female cats at the onset of each session and during preliminary exploration. In three of the five males, $40-\mathrm{Hz}$ activity was observed during neck grip, mount, and pelvic thrusts. A marked decrease in $40-\mathrm{Hz}$ activity occurred during intromission. Two males, however, did not follow this pattern. As soon as the neck grip was secured, all $40-\mathrm{Hz}$ activity disappeared until the female was released. Such $40-\mathrm{Hz}$ activity was also seen in all males during their observation of afterreaction. The females exhibited $40-\mathrm{Hz}$ activity throughout the entire mating session; however, there was a decrease in the incidence of $40-\mathrm{Hz}$ activity during pelvic thrusting just prior to intromission. Higher amplitude activity appeared during intromission and during the afterreaction.

The data from the three males who showed $40-\mathrm{Hz}$ activity during neck grip, mount, pelvic thrust, and observation of after- 
reaction coincided with the observations of Holmes and Egan (1973) and Schwartz and Whalen (1965). The loss of the $40-\mathrm{Hz}$ activity from the onset of neck grip to the release of the neck grip after intromission seen in the other two males could be explained if there was a cessation of airflow through the nasal passage. This airflow appears essential for the appearance of amygdala 40-Hz activity (Gault \& Leaton, 1963; Sheer, 1970). Cessation of airflow could result from the male's burying his head in the neck of the female during the neck grip.

The female data also coincided to those reported by Holmes and Egan (1973). Holmes and Egan suggested that the generally higher amplitude $40-\mathrm{Hz}$ activity seen in the female during intromission was due to a higher state of arousal. However, the present study found that each high-amplitude burst corresponded to the cries emitted by the female during intromission. It should be pointed out that with each cry, a greater amount of air passed through the nasal passage, and this might have precipitated the higher amplitude bursts. In summary, the data suggest that the appearance of amygdala $40-\mathrm{Hz}$ activity during sexual behavior is dependent on airflow and reflects functions of the amygdala in attending to and possibly processing olfactory information.

In conclusion, this study found that attending to environmental cues is highly correlated with specific patterns of electrical activity in the amygdala and the hippocampus. In the male, hippocampal RSA was present during all phases of sexual behavior in which attending to environmental cues aided successful consummation of the sexual responses. In the female, hippocampal RSA, although present during sexual behavior, was less prominent than it was in the male. The $40-\mathrm{Hz}$ amygdala activity, in both the male and female, appeared to be a correlate of orienting to olfactory stimuli, and $40-\mathrm{Hz}$ activity was also apparently dependent on the movement of air through the nasal passages.

\section{REFERENCES}

ADEY, W. R. Neurophysiological correlates of information transaction and storage in brain tissue. In E. Stellar \& J. M. Sprague (Eds.), Progress in physiological psychology (Vol. 1). New York: Academic Press, 1966.

BennetT, T. L. The electrical activity of the hippocampus and processes of attention. In R. L. Isaacson \& K. H. Pribram (Eds.), The hippocampus (Vol. 2). New York: Plenum, 1975.

Bennett, T. L. A gating function for the hippocampus in working memory. Behavioral and Brain Sciences, 1979, 2, 322-323.

BennetT, T. L., \& French, J. Electrical activity of the cat hippocampus during the species-typical gape response: Evidence against the voluntary movement hypothesis. Behavioral Biology, 1977, 21, 432-437.

Delgado, J. M. R., Johnston, V. S., Wallace, J. D., \& BradLeY, R. J. Operant conditioning of amygdala spindling in the free chimpanzee. Brain Research, 1970, 23, 347-362.

GaUlt, F. P., \& LeAton, R. N. Electrical activity of the olfactory system. Electroencephalography and Clinical Neurophysiology, 1963, 15, 299-304.

Green, J. D., \& Arduini, A. A. Hippocampal electrical activity in arousal. Journal of Neurophysiology, 1954, 17, 533-557.

Holmes, E. J., \& EgAN, K. Electrical activity of the cat amygdala during sexual behavior. Physiology \& Behavior, 1973, 10, 863867.

KurTz, R. G. Hippocampal and cortical activity during sexual behavior in the female rat. Journal of Comparative and Physiological Psychology, 1975, 89, 158-169.

Kurtz, R. G., \& Adle R, N. T. Electrophysiological correlates of copulatory behavior in the male rat. Journal of Comparative and Physiological Psychology, 1973, 84, 225-239.

Michael, R. P. Observation upon the sexual behavior of the domestic cat (Felis catus l.) under laboratory conditions. Behaviour, 1961, 18, 1-24.

Schwartz, A. S., \& Whalen, R. E. Amygdala activity during sexual behavior in the male cat. Life Sciences, 1965, 4, 13591366.

SheEr, D. E. Electrophysiological correlates of memory consolidation. In G. Ungar (Ed.), Molecular mechanisms in learning and memory. New York: Plenum, 1970.

Vande Rwolf, C. H., Kramis, R., Gillespie, L. A., \& Bland, B. H. Hippocampal rhythmic slow activity and neocortical low voltage fast activity: Relations to behavior. In $R$. L. Isaacson \& K. H. Pribram (Eds.), The hippocampus (Vol. 2). New York: Plenum, 1975.

(Received for publication March 27, 1982.) 